\title{
DETERMINATION OF TROPOSPHERIC REFRACTION OVER OBSERVATION POINTS IRKM (IRKUTSK), ULAZ (ULAN-UDE) AND BADG (BADARY)
}

\author{
M. G. Dembelov¹, O. F. Lukhneva², A. V. Lukhnev² \\ ${ }^{1}$ Institute of Physical Materials Science, Siberian Branch of RAS, Ulan-Ude, Russia \\ ${ }^{2}$ Institute of the Earth's Crust, Siberian Branch of RAS, Irkutsk, Russia
}

\begin{abstract}
The article describes the possibility of using the passive satellite measurements of the atmosphere to investigate the vertical patterns of pressure, temperature and relative humidity and simulate the altitude dependence of the refractive index of air. The seasonal parameters were determined for the exponential model showing the tropospheric refraction over observation points IRKM (Irkutsk), ULAZ (Ulan-Ude) and BADG (Badary). Post-processing of the input GPS data was conducted to ensure the highest positioning accuracy. In addition to high-precision geodesy, the global positioning method was used for determining the total tropospheric zenith delays (ZTD), which values are used to solve the problems of radio physics and meteorology. The angles of refraction and the true distances were estimated and compared in different seasons of the year. This study shows that the angles of refraction at the observation points located in the Baikal zone do not differ significantly in order of magnitude from the values estimated for other climatic zones.
\end{abstract}

Key words: radio wave refraction; GPS; total tropospheric zenith delay; atmosphere measurements; meteorological data

For citation: Dembelov M.G., Lukhneva O.F., Lukhnev A.V., 2018. Determination of tropospheric refraction over observation points IRKM (Irkutsk), ULAZ (Ulan-Ude) and BADG (Badary). Geodynamics \& Tectonophysics 9 (4), 1205-1215. doi:10.5800/GT-2018-9-4-0391.

Для цитирования: Дембелов М.Г., Лухнева О.Ф., Лухнев А.В. Определение тропосферной рефракции над пунктами наблюдения IRKM (Иркутск), ULAZ (Улан-Удэ) и BADG (Бадары) // Геодинамика и тектонофизика. 2018. T. 9. № 4. C. 1205-1215. doi:10.5800/GT2018-9-4-0391. 


\title{
ОПРЕДЕЛЕНИЕ ТРОПОСФЕРНОЙ РЕФРАКЦИИ НАД ПУНКТАМИ НАБЛЮДЕНИЯ IRKM (ИРКУТСК), ULAZ (УЛАН-УДЭ) И BADG (БАДАРЫ)
}

\author{
М. Г. Дембелов ${ }^{1}$, О. Ф. Лухнева², А. В. Лухнев² \\ ${ }^{1}$ Институт физического материаловедения СО РАН, Улан-Удэ, Россия \\ ${ }^{2}$ Институт земной коры СО РАН, Иркутск, Россия
}

\begin{abstract}
Аннотация: Рассматривается возможность использования пассивной спутниковой локации атмосферы с целью получения данных вертикального распределения давления, температуры и относительной влажности для моделирования высотной зависимости показателя преломления воздуха. Получены сезонные параметры экспоненциальной модели тропосферной рефракции над пунктами наблюдения IRKM (Иркутск), ULAZ (УланУдэ) и BADG (Бадары). В результате постобработки первичных GPS-данных достигается максимальная точность позиционирования. Помимо высокоточной геодезии метод глобального позиционирования позволяет определять полную тропосферную зенитную задержку (ZTD), которая имеет приложения в задачах радиофизики и метеорологии. Выполнены сравнительные расчеты углов рефракции и истинного расстояния в разные сезоны года. Показано несущественное отличие углов рефракции по порядку величин в пунктах наблюдения Байкальской зоны от других климатических зон.
\end{abstract}

Ключевые слова: рефракция радиоволн; GPS; полная тропосферная зенитная задержка; локация атмосферы; метеорологические данные

\section{1. ВВЕДЕНИЕ}

В настоящее время GPS-геодезия является наиболее востребованным и развитым методом изучения современных геодинамических процессов. Для изучения современной геодинамики в Байкальской рифтовой системе продолжается создание сети постоянно действующих пунктов GPS-наблюдений. Пункты наблюдения IRKM (Иркутск), ULAZ (УланУдэ) и BADG (Бадары) являются опорными станциями сети, для которых регулярно определяются современные движения в Байкальском рифте [Lukhnev et al., 2013; San'kov et al., 2003; Sankov et al., 2014]. GPS-станция IRKM расположена на территории Восточно-Сибирского филиала ФГУП «ВНИИФТРИ» в г. Иркутске, станция ULAZ - на территории Института физического материаловедения в г. Улан-Удэ, станция BADG - на территории радиоастрономической обсерватории «Бадары» в Тункинской долине Республики Бурятия. Все перечисленные станции относятся к международной системе пунктов IGS. Попутно в этих же пунктах наблюдения регулярно отбираются данные об ионосферной и тропосферной задержках GPS-сигналов.

Спутники глобальной навигационной системы GPS равномерно распределены в околоземном пространстве. Радиусы спутниковых орбит примерно равны 20000 км. Спутники GPS непрерывно посылают сигналы в направлении земной поверхности, эти сигналы принимаются GPS-приемником. Реальные координаты каждого из спутников определяются с высокой точностью посредством специального принимаемого альманаха. Приемник системы GPS состоит из собственно приемного устройства и малогабаритной антенны с малошумным усилителем (Choke ring antenna). Приемное устройство автономно рассчитывает свои географические координаты и отсечку времени по получаемым от спутников сигналам. В измерениях времени всегда присутствует погрешность, которая обусловлена, в том числе, и влиянием атмосферы. В приемнике вычисляется неточное значение дальности до спутника, или «псевдодальность». Передающая аппаратура спутника излучает сигналы на двух несущих частотах: L1=1575.42 МГц и L2=1227.6 МГц. Навигационные сигналы дополнительно содержат данные об орбитах спутников, информацию о параметрах атмосферы и поправки системного времени. Фазовые наблюдения выполняются для повышения точности измерений.

Погрешности, возникающие при выполнении процедуры GPS-измерений, оказывают существенное влияние на точность определения координат. Наибольшее влияние на процесс распространения навигационных сигналов оказывает верхняя и 
средняя часть атмосферы (ионосфера). Ионосфера представляет собой ионизированный атмосферный слой в диапазоне высот примерно от 50 до 1000 км. Наличие свободных электронов в этом слое вызывает задержку распространения сигнала спутника, которая прямо пропорциональна концентрации электронов и обратно пропорциональна квадрату частоты радиосигнала. Для компенсации ошибки определения псевдодальности, возникающей при этом, используется метод двухчастотных измерений на частотах L1 и L2 (в двухчастотных приемниках) [Afraimovich et al., 2004]. Приемники определяют фазовые задержки GPS-сигналов на обеих частотах.

Тропосфера также оказывает существенное влияние на процесс распространения GPS-сигналов от спутников до приемника. Тропосфера - это самый нижний нейтральный слой атмосферы (до высоты 8-13 км). Высота тропосферы для средних широт составляет около 12 км. Над тропосферой располагается тропопауза, составляющая около 3-4 км. Еще выше находится стратосфера. В тропосфере уровень задержки радиосигнала в первую очередь зависит от метеорологических параметров - атмосферного давления, температуры и влажности воздуха. Кроме того, тропосферная задержка зависит от высоты спутника над горизонтом и отклонения от зенитной нормали. Тропосферная рефракция вызывает отклонение сигнала, который приходит от спутника или другого объекта, находящегося выше тропопаузы. Параметры, связанные с рефракцией в тропосфере, остаются едиными для частот диапазона УКВ.

Процесс тропосферной рефракции хорошо описан в работах Института радиотехники и электроники РАН [Armand, Kolosov, 1965; Bystrov et al., 2009; Moshkov, Pozhidaev, 2014]. В 60-е годы XX века предлагались приближения численного решения интегральной формулы для расчета полной рефракции [Armand, Kolosov, 1965]. В связи с наличием современной вычислительной техники и компьютерных программ в настоящее время подобные интегралы легко вычисляются с большой точностью. В работе [Bystrov et al., 2009] описана рефракция в тропосфере, меняющаяся в соответствии с текущими значениями вертикального распределения показателя преломления. Выполнено сравнение рассчитанных значений полных углов рефракции с экспериментальными данными. Дана оценка расчетов с точностью до 1 угловой секунды для зенитных углов меньше $80^{\circ}$. В работе [Moshkov, Pozhidaev, 2014] pacсмотрены параметры экспоненциальной модели для тропосферной рефракции в условиях Арктики. Выполнены расчеты углов рефракции с использованием точных формул. Показано соотношение между углами истинной и полной рефракции.
Цель настоящей работы заключается в получении новых данных о сезонных параметрах тропосферной рефракции и расчетах углов рефракции в пунктах наблюдения IRKM (Иркутск), ULAZ (УланУдэ) и BADG (Бадары) в разные сезоны года с использованием современной технологии ATOVS, a также в оценке вклада тропосферной задержки в расчет пути прохождения сигнала в тропосфере.

\section{2. ТРОПОСФЕРНАЯ ЗЕНИТНАЯ ЗАДЕРЖКА И ПОКАЗАТЕЛЬ ПРЕЛОМЛЕНИЯ ТРОПОСФЕРЫ}

Тропосферная задержка определяется коэффициентом преломления $n=\sqrt{\varepsilon} \approx 1+(\varepsilon-1) / 2$, где $\varepsilon$ - диэлектрическая проницаемость воздуха, которая изменяется с высотой и близка к единице на уровне границы тропосферы и тропопаузы. Восприимчивость смеси сухого воздуха и водяного пара $k_{\ni}=\varepsilon-1$ подчиняется закону аддитивности, она равна сумме восприимчивости отдельных газов смеси, пропорциональных их парциальным давлениям. Таким образом, ZTD является суммой «сухой» или гидростатической (ZHD) и «влажной» (ZWD) составляющих. Учет «влажной» составляющей по высоте ограничивается верхней частью тропосферы, где может присутствовать водяной пар. Но при расчете полной тропосферной задержки также учитывается нижняя часть стратосферы примерно до 45 км. Величина ZTD является разностью между предполагаемой прямой линией, вдоль которой сигнал распространяется со скоростью света в вакууме, и фактической длиной пути от источника до приемника в зенитном направлении:

$$
\mathrm{ZTD}=\int_{\text {Атмосф }} n(h) d h-\int_{\text {Вакуум }} d h,
$$

где $h$ - переменная по высоте. Иначе формула (1) может быть записана в виде:

$$
\mathrm{ZTD}=\int_{0}^{\infty}(n-1) d h=10^{-6} \int_{0}^{\infty} N(h) d h .
$$

Здесь $N(h)$ - меняющийся с высотой в вертикальном направлении индекс рефракции, который равен: $N(h)=10^{6}(n(h)-1)$. Индекс рефракции также соответствует закону аддитивности согласно известной формуле Фрума-Эссена [Smith, Weintraub, 1953]:

$$
N=(n-1) \cdot 10^{6}=\frac{77.6}{T} p+\frac{3.73 \cdot 10^{5}}{T^{2}} e=N_{T}+N_{e},
$$

где $N_{T}$ - индекс рефракции для сухого воздуха, зависящий от изменений температуры и давления воздуха; $N_{e}-$ индекс рефракции для водяного пара; $T$ - абсолютная температура, К; $p$ - атмосферное 
давление, мбар; $e$ - упругость водяного пара, мбар (1 мбар $=10^{2} \mathrm{H} / \mathrm{M}^{2}$ ). Сухой воздух в тропосфере вносит порядка 1.5-2.5 м в общую задержку сигнала, а водяной пар вносит всего порядка 10-30 см. Формула (3) дает возможность определять индекс рефракции, если известны температура, давление и упругость водяного пара в пункте приема GPSсигналов. Тогда с учетом формулы (1) компоненты полной тропосферной зенитной задержки могут быть выражены посредством интегрирования по вертикальному профилю соответствующих значений индексов рефракции $N_{T}(h)$ и $N_{e}(h)$ [Dembelov et al., 2015, 2016; Lukhneva et al., 2016]:

$$
\begin{aligned}
& \mathrm{ZHD}=10^{-6} \int_{0}^{\infty} N_{T}(h) d h, \\
& \mathrm{ZWD}=10^{-6} \int_{0}^{\infty} N_{e}(h) d h .
\end{aligned}
$$

Как правило, спутники фиксируются в каждый момент времени под определенным углом относительно зенитного направления. Расположение в зенитном направлении является идеальным случаем, когда тропосферная задержка принимает минимальное значение, поэтому реальную тропосферную задержку для линии «спутник - приемник» необходимо выражать в виде:

$$
\mathrm{STD}=\mathrm{ZTD} \cdot m\left(z_{0}\right)
$$

Здесь STD - полная наклонная (slant) тропосферная задержка; $m\left(z_{0}\right)$ - функция отображения (mapping function), которая представляет собой фактор наклона для масштабирования зенитной задержки как функции зенитного угла $\mathrm{z}_{0}$. В настоящее время предложено довольно много примеров функций отображения. Удобно использовать функцию с фиксированными средними коэффициентами, например такую, как функция отображения [Mendes, 1999]:

$$
m\left(z_{0}\right)=\frac{1}{\cos z_{0}+\frac{0.00143}{\operatorname{ctg} z_{0}+0.00035}} .
$$

При зенитных углах менее $60^{\circ}$ можно в качестве функции отображения использовать более простую функцию $m\left(z_{0}\right)=\frac{1}{\cos z_{0}}$.

Первичные данные, полученные в процессе измерений на постоянных GPS-станциях, обрабатывались пакетом программ GAMIT, разработанных в Массачусетском технологическом институте [King, Bock, 1999]. На рис. 1 показаны обработанные данные изменения полной ZTD для постоянных пунктов наблюдения IRKM (Иркутск), ULAZ (Улан-Удэ), BADG (Бадары) и ULAB (Улан-Батор) за весь 2016 г. Данные по ZTD получены посредством постоянных
GPS-измерений. Разные годовые уровни тропосферных задержек объясняются разной высотой расположения над уровнем моря приемных антенн пунктов GPS-наблюдения (IRKM - 496 м, ULAZ - 514 м, BADG - 838 м, ULAB - 1599 м). Таким образом, чем выше местоположение пункта наблюдения над уровнем моря, тем меньшие слои тропосферы учитываются при определении зенитной задержки и тем меньше зенитная задержка сигнала.

\section{3. ВЕРТИКАЛЬНЫЕ ПРОФИЛИ ИНДЕКСА РЕФРАКЦИИ}

Современные космические технологии позволяют подробно исследовать атмосферу, а использование инструментального комплекса ATOVS на полярно-орбитальных спутниках NOAA дает возможность восстанавливать вертикальные профили температуры и влажность воздуха на разных уровнях атмосферного давления с пространственным разрешением 20 км в инфракрасном диапазоне и 50 км в волновом диапазоне [Grigoriev, Kashkin, 2013; Kashkin, 2014]. При отсутствии облачности сканирующий спектрофотометр, входящий в комплекс ATOVS, позволяет определять профили метеорологических данных до высоты 40 км над поверхностью Земли. Обработка первичных данных ATOVS выполняется с помощью программного продукта AAPP [Kashkin, 2014]. При зондировании тропосферы и стратосферы используется зависимость ширины линии излучения от давления, поэтому данные распределения температуры и влажности привязаны не к высоте, а к атмосферному давлению. В результате выдается вертикальное распределение температуры и влажности на разных уровнях атмосферного давления на сетке $1^{\circ} \times 1^{\circ}$ по широте и долготе. В настоящее время высотные метеоданные, получаемые в результате запусков радиозондов, являются наиболее достоверными для подробного изучения нижней части атмосферы.

На рис. 2, a, б, представлены сравнительные графики измеренных вертикальных профилей температуры, полученных для зимних и летних условий над пунктами наблюдения Ангарск (по данным запусков радиозондов Аэрологической службой Росгидромета) и Иркутск (по данным дистанционного зондирования спутниками NOAA). Несмотря на дистанционный разнос двух пунктов наблюдения (около 43 км) [Lukhneva et al., 2016], можно наблюдать достаточно хорошее соответствие полученных результатов, которые могут быть использованы для определения рефракционных параметров тропосферы.

Зависимость индекса рефракции $(N)$ от высоты $h$ можно записать в экспоненциальном виде [Moshkov, Pozhidaev, 2014]: 




Рис. 1. Годовой ход полных тропосферных зенитных задержек для пунктов наблюдения ULAZ (Улан-Удэ), IRKM (Иркутск), BADG (Бадары) и ULAB (Улан-Батор), полученных по GPS-измерениям в 2016 г.

Fig. 1. The annual pattern of total tropospheric zenith delays for observation points ULAZ (Ulan-Ude), IRKM (Irkutsk), BADG (Badary) and ULAB (Ulan Bator), according to GPS measurements of 2016.

$$
N(h)=(n-1) \times 10^{6}=N_{0} \exp (-\beta h),
$$

где $N_{0}$ - индекс рефракции на поверхности Земли; $\beta$, км ${ }^{-1}$ - скорость убывания индекса рефракции по высоте $h$, км. Для этих же дат на рис. 3 , представлены сравнительные графики индексов рефракции, полученных посредством спутникового зондирования NOAA и расчета по экспоненциальной модели (8), над г. Иркутском. Для экспоненциальной модели значения $N_{0}$ и $\beta$ выбраны равными соответственно 298 и 0.135 для февраля и 316 и 0.136 для июля 2017 г. По спутниковым данным индекс рефракции $N$ на разных высотах рассчитывался по формуле Фрума-Эссена (3).

В таблице 1 приведены результаты аппроксимации параметров рефракции $\left(N_{0}\right.$ и $\left.\beta\right)$ экспоненциальной моделью с применением метода наименьших квадратов по результатам вертикального зондирования спутниками NOAA в разное время 2017 г. Приведенные в таблице 1 параметры являются характерными для четырех сезонов года и служат ключевыми входными данными для расчета углов рефракции, истинного расстояния и поправки для расстояния, вызванной рефракционным эффектом.

\section{4. РАСЧЕТ ПАРАМЕТРОВ РЕФРАКЦИИ}

Измеренная STD учитывает преломление сигнала в нижней нейтральной части атмосферы. Полученные посредством GPS-измерений значения полной ZTD могут быть использованы для изучения истинного положения излучающего объекта в диапазоне УКВ, расстояния до него от направленной антенны, расположенной на поверхности Земли, а также всех углов рефракции (рис. 4).

Параметры, связанные с рефракцией: А - место расположения приемника; В - истинная точка над тропосферой; B' - кажущаяся за счет рефракции точка над тропосферой; $R$ - радиус Земли; $L$ - расстояние по прямой; $L^{\prime}$ - кажущееся расстояние; $H$ высота точки В над Землей; $\mathrm{z}_{0}$ - видимый зенитный угол; $\alpha-$ угол полной рефракции; $\delta$ - угол истинной рефракции; $\psi$ - угол фотограмметрической рефракции $(\psi=\alpha-\delta) ; \theta$ - центральный угол. Для расчета угла полной рефракции $\alpha$ и центрального угла $\theta$ удобно использовать формулы [Moshkov, Pozhidaev, 2014]:

$$
\alpha=-n_{0} \sin z_{0} \int_{0}^{H} \frac{\left(\frac{d n(h)}{d h}\right) d h}{n(h) \sqrt{n(h)^{2}(1+h / R)^{2}-n_{0}^{2} \sin ^{2} z_{0}}},
$$


M.G. Dembelov et al.: Determination of tropospheric refraction...
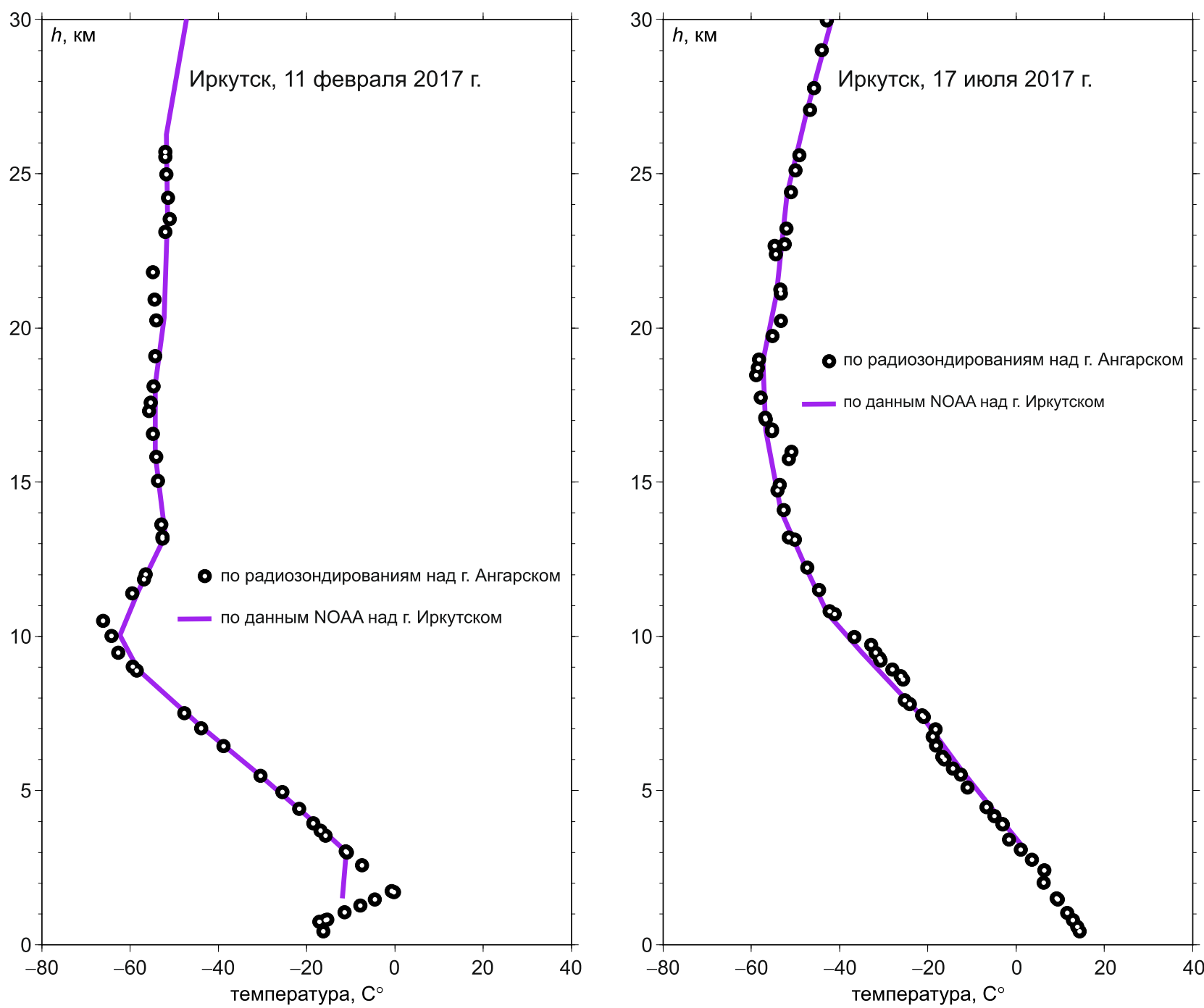

Рис. 2. Сравнение высотных температурных профилей, полученных методами радиозондирования и спутникового зондирования 11 февраля 2017 г. и 17 июля 2017 г.

Fig. 2. Comparison of altitude temperature profiles obtained by radio sounding and satellite sounding methods on February 11, 2017 and July 17, 2017.

$$
\theta=-n_{0} \sin z_{0} \int_{0}^{H} \frac{d h}{(R+h) \sqrt{n(h)^{2}(1+h / R)^{2}-n_{0}^{2} \sin ^{2} z_{0}}}
$$

Здесь $n_{0}$ - показатель преломления у земной поверхности, $n(h)$ - высотный профиль показателя преломления тропосферы (см. формулу (2)), $\frac{d n(h)}{d h}-$ вертикальный градиент показателя преломления.

Расстояние $L$ можно вычислить по теореме косинусов, зная центральный угол $\theta$. Тогда легко определяется угол $\angle \mathrm{BAO}$, откуда вычисляется угол $\delta=\pi-z_{0}-\angle$ ВАО. Разница $\Delta L=L^{\prime}-L$, которая представляет собой тропосферную задержку, может быть наиболее точно определена по данным GPS-измерений. Значение $\Delta L$ составляет очень малую величину относительно расстояния $L$. Даже для расстояния $L=50$ км при $\mathrm{z}_{0}=60^{\circ}$ значение $\Delta L$ не превышает 0.01 \%. Таким образом, для высот $H$, больших 50 км, используя значения STD, полученные посредством GPS-измерений, можно определить путь прохождения сигнала с учетом рефракции $L^{\prime}$, поскольку для таких высот и выше угол полной рефракции и тропосферная задержка практически не изменяются.

Для расчета углов $\alpha$ и $\theta$ по формулам (9) и (10) составлена и протестирована программа на алгоритмическом языке Фортран-77. В таблице 2 приведены результаты расчетов углов полной и истинной рефракции, а также дальности от приемной антенны до излучающих объектов на разных высотах над Землей. Приведенные значения углов $\delta$ являются поправкой к зенитному углу: $z_{0}^{\prime}=z_{0}+\delta$. Также можно определить величину расхождения между кажущейся и истинной точками объекта 

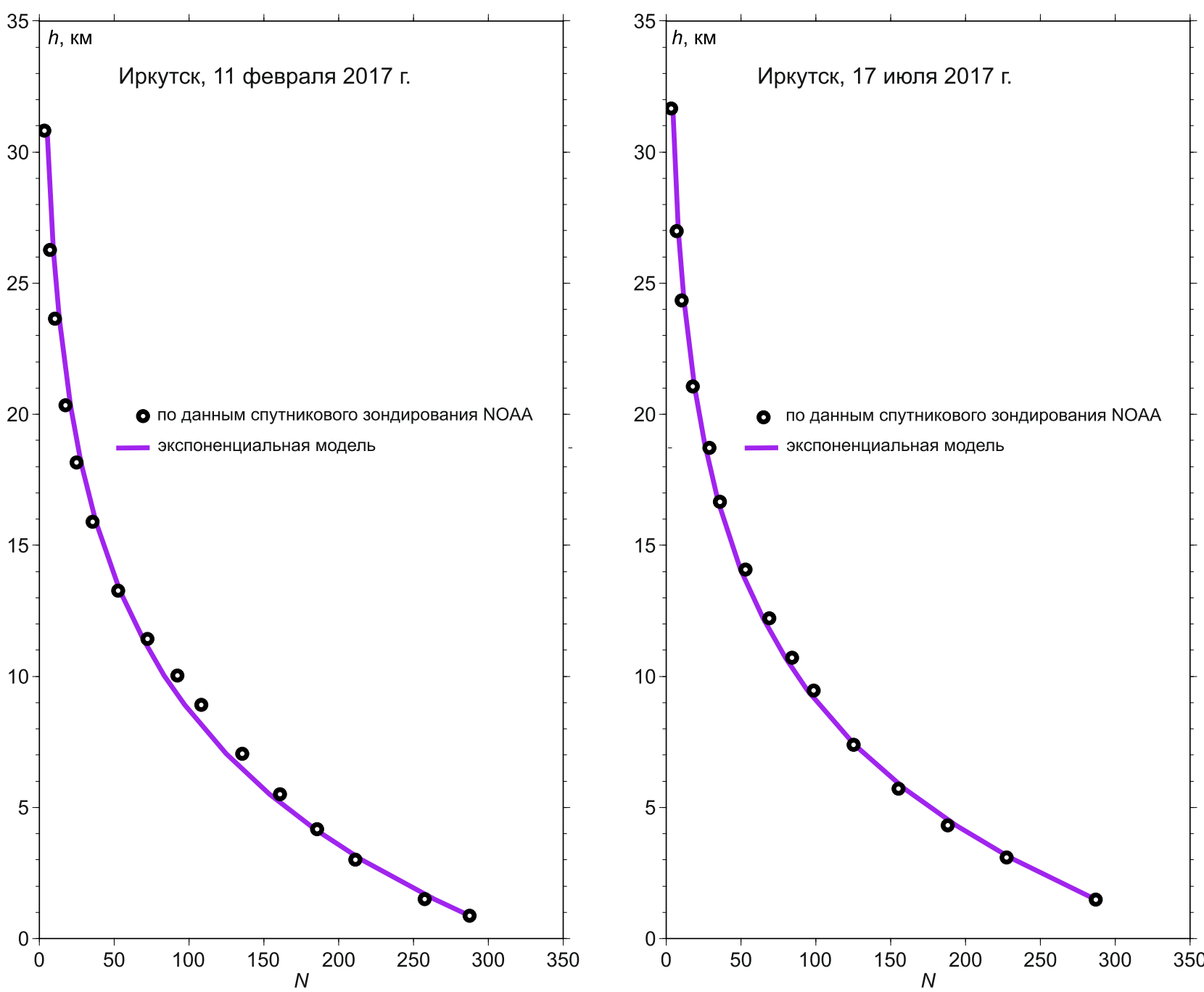

Рис. 3. Сравнительные высотные профили индекса рефракции над г. Иркутском.

Fig. 3. Comparative altitude profiles of the refraction index over the city of Irkutsk.

T а б л и ц а 1. Параметры рефракции для трех пунктов наблюдения в разные сезоны года $\mathrm{T}$ a b l e 1 . Refraction parameters for the three observation points in different seasons

\begin{tabular}{|c|c|c|c|}
\hline Пункты наблюдения & Месяцы & $N_{0}$ & $\beta$, км$^{-1}$ \\
\hline $\begin{array}{l}\text { 1. Иркутск (IRKM) } \\
\text { (52.2228 с.ш., } 104.3183 \text { в.д.) } \\
h=496 \text { м }\end{array}$ & $\begin{array}{l}\text { февраль } \\
\text { апрель } \\
\text { июль } \\
\text { октябрь }\end{array}$ & $\begin{array}{l}298 \\
288 \\
316 \\
297\end{array}$ & $\begin{array}{l}0.135 \\
0.134 \\
0.136 \\
0.133\end{array}$ \\
\hline $\begin{array}{l}\text { 2. Улан-Удэ (ULAZ) } \\
\text { (51.8144 с.ш., 107.6219 в.д.) } \\
h=514 \text { м }\end{array}$ & $\begin{array}{l}\text { февраль } \\
\text { апрель } \\
\text { июль } \\
\text { октябрь }\end{array}$ & $\begin{array}{l}294 \\
287 \\
310 \\
292\end{array}$ & $\begin{array}{l}0.134 \\
0.134 \\
0.135 \\
0.132\end{array}$ \\
\hline $\begin{array}{l}\text { 3. Бадары (BADG) } \\
\text { (51.7697 с.ш., 102.2347 в.д.) } \\
h=838 \text { м }\end{array}$ & $\begin{array}{l}\text { февраль } \\
\text { апрель } \\
\text { июль } \\
\text { октябрь }\end{array}$ & $\begin{array}{l}289 \\
277 \\
308 \\
287\end{array}$ & $\begin{array}{l}0.135 \\
0.136 \\
0.144 \\
0.134\end{array}$ \\
\hline
\end{tabular}




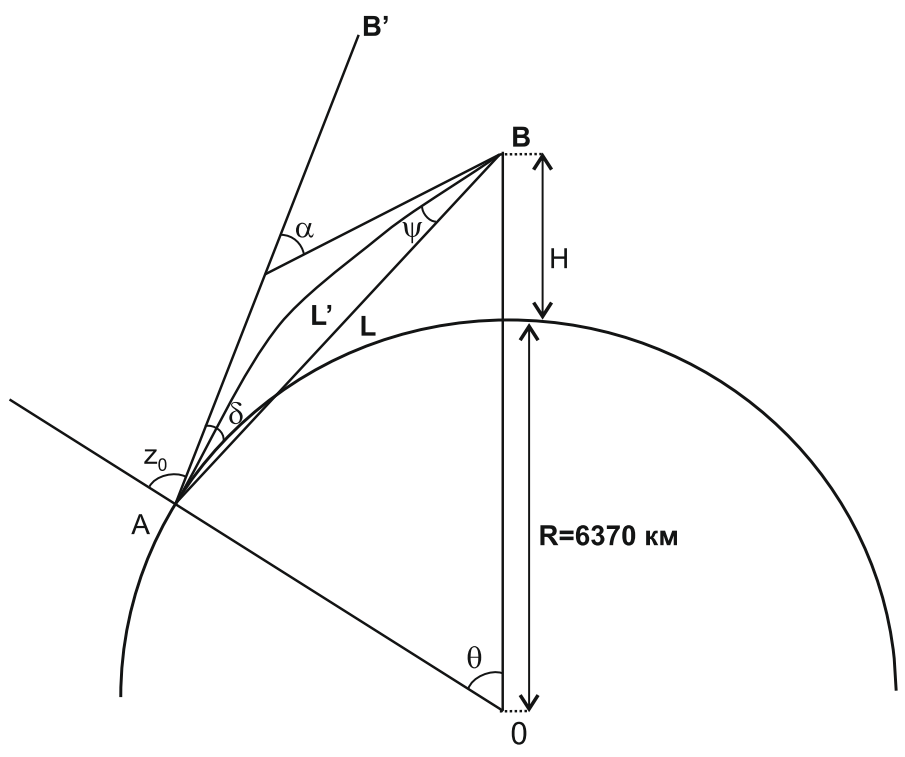

Рис. 4. Геометрия задачи о тропосферной рефракции.

Fig. 4. Geometry of the problem of tropospheric refraction.

T а б л и ц а 2. Углы рефракции и истинная дальность между приемной антенной и передающим объектом, находящимся на высотах 15, 50 и 100 км, для видимых углов 60 и 75 в разные сезоны года

$\mathrm{T}$ a $\mathrm{b} l$ e 2 . The angles of refraction and the real distance between the receiving antenna and the transmitter located at heights of 15,50 and $100 \mathrm{~km}$, for visible angles of $60^{\circ}$ and $75^{\circ}$ in different seasons

\begin{tabular}{|c|c|c|c|c|c|c|c|c|}
\hline \multirow[t]{2}{*}{ Пункты наблюдения } & \multirow[t]{2}{*}{ Месяцы } & \multirow[t]{2}{*}{ Н, км } & \multicolumn{3}{|l|}{$\mathrm{z}_{0}=60^{\circ}$} & \multicolumn{3}{|l|}{$\mathrm{z}_{0}=75^{\circ}$} \\
\hline & & & $\alpha$, угл. с. & $\delta$, угл. с. & $\mathrm{L}, \mathrm{KM}$ & $\alpha$, угл. с. & $\delta$, угл. с. & L, KM \\
\hline \multirow[t]{9}{*}{ 1. Иркутск (IRKM) } & \multirow[t]{2}{*}{ февраль } & 15 & 92 & 61 & 29.9 & 197 & 130 & 57 \\
\hline & & 100 & 106 & 98 & 196 & 226 & 208 & 352 \\
\hline & \multirow[t]{2}{*}{ апрель } & 15 & 89 & 58 & 29.9 & 190 & 125 & 57 \\
\hline & & 50 & 102 & 87 & 99 & 218 & 185 & 184 \\
\hline & \multirow{2}{*}{ июль } & 50 & 112 & 96 & 99 & 240 & 204 & 184 \\
\hline & & 100 & 112 & 104 & 196 & 240 & 221 & 353 \\
\hline & \multirow[t]{3}{*}{ октябрь } & 15 & 91 & 60 & 29.9 & 195 & 128 & 57 \\
\hline & & 50 & 105 & 90 & 99 & 225 & 191 & 184 \\
\hline & & 100 & 106 & 98 & 196 & 225 & 207 & 353 \\
\hline \multirow[t]{8}{*}{ 2. Улан-Удэ (ULAZ) } & февраль & 15 & 91 & 60 & 29.9 & 194 & 127 & 57 \\
\hline & апрель & 100 & 102 & 94 & 196 & 218 & 200 & 353 \\
\hline & \multirow[t]{3}{*}{ июль } & 15 & 96 & 63 & 29.9 & 205 & 135 & 57 \\
\hline & & 50 & 110 & 94 & 99 & 235 & 200 & 184 \\
\hline & & 100 & 110 & 102 & 196 & 235 & 217 & 353 \\
\hline & \multirow[t]{3}{*}{ октябрь } & 15 & 90 & 59 & 29.9 & 192 & 126 & 57 \\
\hline & & 50 & 104 & 88 & 99 & 221 & 187 & 184 \\
\hline & & 100 & 104 & 96 & 196 & 221 & 204 & 353 \\
\hline \multirow[t]{5}{*}{ 3. Бадары (BADG) } & \multirow[t]{3}{*}{ февраль } & 15 & 89 & 59 & 29.9 & 191 & 126 & 57 \\
\hline & & 50 & 103 & 88 & 99 & 219 & 186 & 184 \\
\hline & & 100 & 103 & 95 & 196 & 219 & 202 & 353 \\
\hline & \multirow[t]{2}{*}{ апрель } & 15 & 86 & 57 & 29.9 & 184 & 121 & 57 \\
\hline & & 50 & 98 & 84 & 99 & 210 & 177 & 184 \\
\hline
\end{tabular}


Т а б л и ц а 3. Углы полной рефракции и тропосферные задержки для видимых углов 80 и $87^{\circ}$ в разные сезоны года

$\mathrm{T}$ a b l e 3 . The angles of total refraction and the tropospheric delays for visible angles of $80^{\circ}$ and $87^{\circ}$ in different seasons

\begin{tabular}{|c|c|c|c|c|c|}
\hline \multirow[t]{2}{*}{ Пункты наблюдения } & \multirow[t]{2}{*}{ Месяцы } & \multicolumn{2}{|l|}{$\mathrm{z}_{0}=80^{\circ}$} & \multicolumn{2}{|l|}{$\mathrm{z}_{0}=87^{\circ}$} \\
\hline & & $\alpha$, угл. с & STD, $\mathrm{M}$ & $\alpha$, угл. с & STD, $\mathrm{M}$ \\
\hline \multirow[t]{3}{*}{ 1. Иркутск (IRKM) } & февраль & 338 & 12.5 & 940 & 28.6 \\
\hline & июль & 358 & 12.8 & 999 & 29.3 \\
\hline & октябрь & 337 & 12.5 & 934 & 28.5 \\
\hline \multirow[t]{2}{*}{ 2. Улан-Удэ (ULAZ) } & февраль & 333 & 12.3 & 925 & 28.1 \\
\hline & октябрь & 331 & 12.3 & 910 & 28.1 \\
\hline \multirow[t]{4}{*}{ 3. Бадары (BADG) } & февраль & 328 & 12.2 & 910 & 27.7 \\
\hline & апрель & 314 & 11.9 & 873 & 27.2 \\
\hline & июль & 350 & 12.4 & 983 & 28.3 \\
\hline & октябрь & 325 & 12.0 & 903 & 27.5 \\
\hline
\end{tabular}

излучения: $S=L \cdot \delta$. Например, для г. Иркутска в летнее время для зенитного угла $z_{0}=60^{\circ}$ и при высоте источника 15 км расхождение равно 14 м, а для зенитного угла $z_{0}=75^{\circ}$ и при высоте источника 100 км такое расхождение равно 371 м.

При стремлении кажущегося источника ближе к горизонту, когда видимый угол $z_{0}$ стремится к $90^{\circ}$, значения углов полной рефракции и тропосферной задержки STD увеличиваются быстрее. В таблице 3 приведены результаты расчетов углов полной рефракции при $H \rightarrow \infty$ и тропосферных задержек в разные сезоны года. Наблюдается синхронное сезонное изменение значений $\alpha$ и STD. Отметим, что значения STD в целом не имеют существенной вариативности в течение года. Так, для пунктов IRKM и ULAZ отличие летних значений STD от весенних составляет примерно $3.5 \%$, а для пункта BADG примерно $4 \%$.

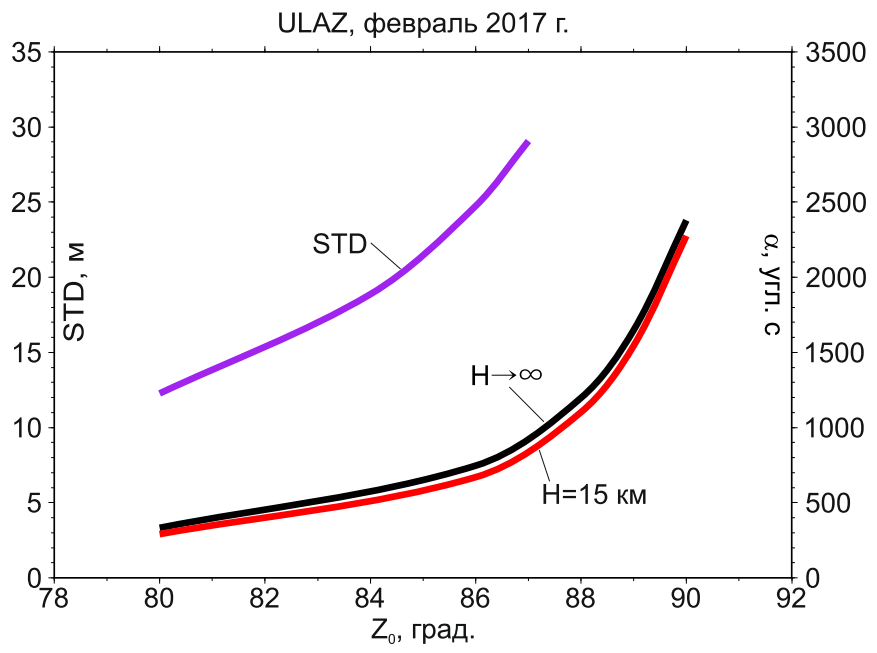

На рис. 5 показаны изменения величины $\alpha$, рассчитанные для пункта ULAZ в зимний сезон при высоте $H=15$ км и $H \rightarrow \infty$, в зависимости от видимых углов $z_{0}$, близких к $90^{\circ}$. Можно наблюдать отличие значений $\alpha$ при $H \rightarrow \infty$ от значений при $H=15$ км на $12 \%$ для $z_{0}=80^{\circ}$ и на $6 \%$ для $z_{0}=89^{\circ}$. Также расчеты показали, что отличия значений $\alpha$ при $H \rightarrow \infty$ от значений при $H$ больше 40 км практически отсутствуют. Здесь же показан график изменения величины STD в зависимости от $z_{0}$.

В задаче о рефракции истинное расстояние $L$ является одним из искомых результатов. Согласно полученным результатам, показанным в таблице 2, величина $L$ рассчитывается довольно точно. Разброс данных для всех пунктов наблюдения в разные сезоны года находился в пределах $0.2 \%$. Расчеты показали, что угол полной рефракции $\alpha$ растет до высоты нижней части стратосферы, где воз-

Рис. 5. Графики изменения величины угла полной рефракции и тропосферной задержки пункта наблюдения ULAZ (Улан-Удэ) в феврале 2017 г. для высот источника $H=15$ и $H \rightarrow \infty$ при видимых зенитных углах $z_{0}$, близких к $90^{\circ}$.

Fig. 5. Changes in the angle of total refraction and the tropospheric delays over observation point ULAZ (UlanUde) in February 2017. Height of the source: $H=15$, and $H \rightarrow \infty$; visible zenith angles $Z_{0}$ close to $90^{\circ}$. 
можно присутствие водяного пара, затем при увеличении высоты значения угла $\alpha$ практически не изменяются. Сравнение изменения значений STD, полученных посредством GPS-измерений, с рассчитанными величинами углов полной рефракции в зависимости от видимых зенитных углов $z_{0}$ в разные сезоны года показало возможность моделирования геометрических величин рефракции с дополнительным использованием GPS-измерений.

\section{5.ЗАКЛЮЧЕНИЕ}

Использование данных полярно-орбитальных спутников NOAA по вертикальным профилям температуры и влажности воздуха на разных уровнях атмосферного давления позволяет успешно восстанавливать вертикальные профили индекса рефракции, которые служат исходными данными для расчета углов рефракции. Получены характерные усредненные месячные данные параметров экспоненциальной модели $\left(N_{0}\right.$ и $\left.\beta\right)$ для пунктов наблюдения Иркутск (IRKM), Улан-Удэ (ULAZ) и Бадары (BADG) в разные сезоны года.

Составлена и опробована программа расчета углов рефракции сигналов, испытывающих влияние тропосферы. Показана возможность расчета всех основных геометрических параметров рефракции (углы рефракции, истинное и кажущееся расстояние) с использованием формул (9) и (10).

Выполнены расчеты углов рефракции и истинной дальности от наземной антенны до излучающего объекта на разных высотах в разные сезоны года над пунктами наблюдения IRKM, ULAZ и BADG. Расчеты показали, что углы полной рефракции при высоте точки излучения выше 40 км практически не меняются.

Показано, что зондирование тропосферы методом GPS-измерений позволяет исследовать рефракционные эффекты как сигналов GPS, так и других сигналов диапазона УКВ.

\section{6. ЛИТЕРАTУРA / REFERENCES}

Afraimovich E.L., Bashkuev Yu.B., Berngardt O.I., Gatsutsev A.V., Dembelov M.G., Shpynev B.G., Kobzar' V.A., Kushnarev D.S., Musin V.Yu., Pushkin P.Yu., Perevalova N.P., 2004. Detection of traveling ionospheric disturbances from the data of simultaneous measurements of the electron concentration, total electron content, and doppler frequency shift at the ISTP radiophysical complex. Geomagnetism and Aeronomy 44 (4), 463-475.

Armand N.A., Kolosov M.A., 1965. On the refraction of radio waves in the troposphere. Radiotekhnika i Elektronika (Radio Engineering and Electronics) 10 (8), 1401-1409 (in Russian) [Арманд Н.А., Колосов М.А. О рефракции радиоволн в тропосфере // Радиотехника и электроника. 1965. Т. 10. № 8. С. 1401-1409].

Bystrov R.P., Sokolov A.V., Sokolov S.A., 2009. Influence of refraction on the absorption of millimeter waves. In: Radiolocation and radio communication. Proceedings of the $3^{\text {rd }}$ All-Russia Scientific Conference. IRE RAS, Moscow, p. 655-660 (in Russian) [Быстров Р.П., Соколов А.В., Соколов С.А. Влияние рефракции на поглощение миллиметровых волн // Радиолокация и радиосвязь: Труды III Всероссийской научной конференции. М.: ИРЭ PAH, 2009. C. 655-660].

Dembelov M.G., Bashkuev Yu.B., Lukhnev A.V., Lukhneva O.F., San'kov V.A., 2015. Diagnostics of atmospheric water vapor content according to GPS measurements. Atmospheric and Oceanic Optics 28 (4), 291-296. https://doi.org/ 10.1134/S1024856015040053.

Dembelov M.G., Bashkuev Yu.B., Lukhnev A.V., Lukhneva O.F., San'kov V.A., 2016. The moisture content in the troposphere in the Baikal region from GPS measurements. Zhurnal Radioelektroniki (Journal of Radio Electronics) (3), 1-16 (in Russian) [Дембелов М.Г., Башкуев Ю.Б., Лухнев А.В., Лухнева О.Ф., Саньков В.А. Влагосодержание тропосферы в Байкальском регионе по данным GPS-измерений // Журнал радиоэлектроники. 2016. № 3. C. 1-16]. Available from: http://jre.cplire.ru/jre/mar16/10/text.pdf.

Grigoriev A.S., Kashkin V.B., 2013. Detection of ionosphere and troposphere response to seismic activity according to satellite data. Siberian Journal of Science and Technology (5), 152-156 (in Russian) [Григорьев А.С., Кашкин В.Б. Обнаружение ионосферных и тропосферных откликов сейсмической активности по спутниковым данным // Сибирский журнал науки и технологий. 2013. № 5. С. 152-156].

Kashkin V.B., 2014. Internal gravity waves in the troposphere. Atmospheric and Oceanic Optics 27 (1), 1-9. https:// doi.org/10.1134/S1024856014010059.

King R.W., Bock Y., 1999. Documentation for the GAMIT GPS Analysis Software, Version 9.9. Massachusetts Institute of Technology, Cambridge. Available from: http://geoweb.mit.edu/gg/docs.php.

Lukhnev A.V., San'kov V.A., Miroshnichenko A.I., Ashurkov S.V., Byzov L.M., San'kov A.V., Bashkuev Yu.B., Dembelov M.G., Calais E., 2013. GPS-measurements of recent crustal deformation in the junction zone of the rift segments in the central Baikal rift system. Russian Geology and Geophysics 54 (11), 1417-1426. https://doi.org/10.1016/j.rgg. 2013.10.010.

Lukhneva O.F., Dembelov M.G., Lukhnev A.V., 2016. The determination of atmospheric water content from meteorological and GPS data. Geodynamics \& Tectonophysics 7 (4), 545-553. https://doi.org/10.5800/GT-2016-7-4-0222. 
Mendes V., 1999. Modeling the Neutral Atmospheric Propagation Delay in Radiometric Space Techniques. University of New Brunswick, Brunswick, 353 p.

Moshkov A.V., Pozhidaev V.N., 2014. Features of radio wave refraction in the near-polar regions. Journal of Communications Technology and Electronics 59 (11), 1107-1111. https://doi.org/10.1134/S1064226914110163.

San'kov V.A., Lukhnev A.V., Miroshnichenko A.I., Levi K.G., Ashurkov S.V., Bashkuev Yu.B., Dembelov M.G., Calais E., Déverchère J., Vergnolle M., Bechtur B., Amarjargal Ch., 2003. Recent movements of the Earth's crust in the MongolSiberian region inferred from GPS geodetic data. Doklady Earth Sciences 393 (8), 1082-1085.

Sankov V.A., Lukhnev A.V., Miroshnitchenko A.I., Dobrynina A.A., Ashurkov S.V., Byzov L.M., Dembelov M.G., Calais E., Déverchère J., 2014. Contemporary horizontal movements and seismicity of the south Baikal basin (Baikal rift system). Izvestiya, Physics of the Solid Earth 50 (6), 785-794. https://doi.org/10.1134/S106935131406007X.

Smith E.K., Weintraub S., 1953. The constants in the equation for atmospheric refractive index at radio frequencies. Proceedings of the IRE 41 (8), 1035-1037. https://doi.org/10.1109/JRPROC.1953.274297.

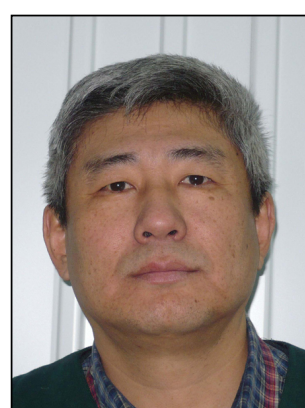

Михаил Георгиевич Дембелов, канд. физ.-мат. наук, с.н.с.

Институт физического материаловедения СО РАН

670047, Улан-Удэ, ул. Сахьяновой, 6, Россия

e-mail: mdembelov@mail.ru

ORCID ID https://orcid.org/0000-0002-7281-4252

Mikhail G. Dembelov, Candidate of Physics and Mathematics, Senior Researcher Institute of Physical Materials Science, Siberian Branch of RAS

6 Sakhyanova street, Ulan-Ude 670047, Russia

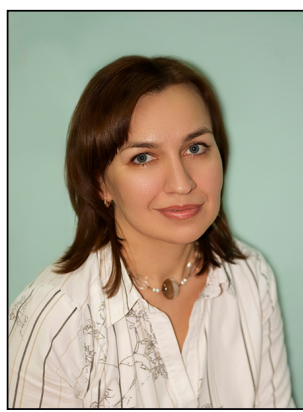

Ольга Федоровна Лухнева, канд. геол.-мин. наук, н.с.

Институт земной коры СО РАН

664033, Иркутск, ул. Лермонтова, 128, Россия

e-mail: olgal@crust.irk.ru

ORCID ID https://orcid.org/0000-0001-9930-1871

Olga F. Lukhneva, Candidate of Geology and Mineralogy, Researcher Institute of the Earth's Crust, Siberian Branch of RAS

128 Lermontov street, Irkutsk 664033, Russia

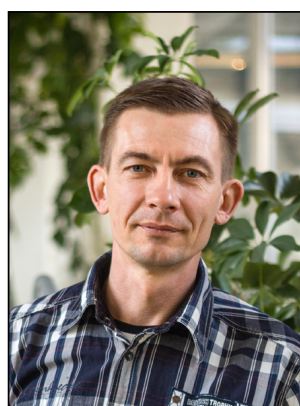

Андрей Викторович Лухнев, канд. геол.-мин. наук, с.н.с.

Институт земной коры СО РАН

664033, Иркутск, ул. Лермонтова, 128, Россия

e-mail: loukhnev@crust.irk.ru

ORCID ID https://orcid.org/0000-0003-1599-5373

Andrei V. Lukhnev, Candidate of Geology and Mineralogy, Senior Researcher Institute of the Earth's Crust, Siberian Branch of RAS

128 Lermontov street, Irkutsk 664033, Russia 